\title{
Isolation and Characterization of Seneca Valley Virus Spread from Pig to Mink
}

\author{
Chao Chen ${ }^{1}$, Zida $\mathrm{Nai}^{1}$, Yao Wang ${ }^{1}$, Ziliang Qin ${ }^{1}$, Qinjian Niu ${ }^{1}$, Yuwen $\mathrm{Li}^{1}$, Yaguang \\ $\mathrm{Tian}^{1}$, Yuhui $\mathrm{Ma}^{2}$, Xinmiao $\mathrm{He}^{3}$, Di Liu ${ }^{3}$, and Xinpeng Jiang ${ }^{1}$ \\ ${ }^{1}$ Northeast Agricultural University \\ ${ }^{2}$ Animal Husbandry and Veterinary Development Center of Zhaosu \\ ${ }^{3}$ Heilongjiang Academy of Agricultural Sciences
}

April 18, 2021

\begin{abstract}
Seneca Valley Virus (SVV) infection has recently spread to pig farms in Canada, America, and China. Humans, mice, and houseflies have been identified as hosts and reservoirs. Although such cross-species transmission events are often limited, sustained outbreaks in a new mammalian host can occur. To determine if mink are a new mammalian host of SSV, we studied the molecular characteristics of isolated SVV genomes and analyzed challenge, pathology, and immune response data. The study was the first systemic analysis of a newly isolated strain of SVV from pigs. The strain caused an intestinal infection with associated pathologic changes in mink. SVV stimulated the production of a specific neutralizing antibody. The findings highlight the importance of identifying SVV infection in mink and the host to detect mutated SSV that could threaten livestock and pose public health and economic risks.
\end{abstract}

Original article

Isolation and Characterization of Seneca Valley Virus Spread from Pig to Mink

Chao Chen ${ }^{1}$, Zida $\mathrm{Nai}^{1}$, Yao Wang ${ }^{1}$, Ziliang Qin ${ }^{1}$, Qinjian Niu ${ }^{1}$, Yuwen $\mathrm{Li}^{1}$, Yaguang Tian ${ }^{1,3}$, Yuhui $\mathrm{Ma}^{4}$, Xinmiao $\mathrm{He}^{2}$,Di Liu ${ }^{1,2^{*}}$, Xinpeng Jiang, ${ }^{1, *}$

1. Northeast Agricultural University, Harbin, Heilongjiang 150030, P. R. China

2. Key Laboratory of Combining Farming and Animal Husbandry, Ministry of Agriculture, Animal Husbandry Research Institute, Heilongjiang Academy of Agricultural Sciences No. 368 Xuefu Road, Harbin 150086, P. R. China

3. Undergraduate Experimental and Teaching Center, College of Animal Science and Technology, Northeast Agricultural University, Harbin, Heilongjiang 150030, P. R. China.

4. Animal Husbandry and Veterinary Development Center of Zhaosu,Xinjiang 835600, P. R. China.

* Corresponding author. Tel.: +86-451-55190722

E-mail address: jiangxinpeng@neau.edu.cn (Xinpeng. Jiang)

liudi1963@163.com(Di Liu)

\section{Summary}


Seneca Valley Virus (SVV) infection has recently spread to pig farms in Canada, America, and China. Humans, mice, and houseflies have been identified as hosts and reservoirs. Although such cross-species transmission events are often limited, sustained outbreaks in a new mammalian host can occur. To determine if mink are a new mammalian host of SSV, we studied the molecular characteristics of isolated SVV genomes and analyzed challenge, pathology, and immune response data. The study was the first systemic analysis of a newly isolated strain of SVV from pigs. The strain caused an intestinal infection with associated pathologic changes in mink. SVV stimulated the production of a specific neutralizing antibody. The findings highlight the importance of identifying SVV infection in mink and the host to detect mutated SSV that could threaten livestock and pose public health and economic risks.

Keywords: Seneca Valley Virus, Cross-host transmission, Mink

\section{INTRODUCTION}

Picornaviruses commonly infect a wide range of animal species, including humans. They cause a broad range of clinical symptoms, such as myocarditis, meningitis, encephalitis, diarrhea, and paralysis. Recent research has focused on the dependency between host transmission and intestinal picornavirus infections. Many reports have demonstrated that the picornavirus that can overcome host barriers have close genetic similarities between species, and that different viral genera can adapt to new hosts; examples include Kobuvirus, Foot-and-mouth disease virus, and Enterovirus(Mombo et al., 2015; Oberste et al., 2013; Omondi et al., 2019). SVV is a single-stranded positive-sense RNA virus that belongs to the genus Senecavirus within the family Picornaviridae. SVV is closely related to members of the genus Cardiovirus, which infected a wide range of vertebrate animals, including pigs, mice, and humans. Histopathological examinations have revealed that SVV causes pathological changes in epithelial cells and acute lameness myocarditis, vesicular lesions, interstitial pneumonia, and atrophy of intestinal villi with vacuolation of the superficial epithelial cells(Canning et al., 2016; Leme, Oliveira, Alfieri, Headley, \& Alfieri, 2016). SVV can spread to other internal organs without any other clinical manifestation.

In cross-species transmission, the concept of "sentinel species" is important in public health science. The use of a sentinel species can provide the best animal model for SVV research. Such a model provides integrated and relevant information on virus evolution through adaptive mutations and neofunctionalization(Bo-Shun et al., 2020; Liu et al., 2020). The mink (Mustela vison ) is a member of the weasel family. It is a carnivorous mammal that has a high trophic status in the wild. The mink diet is varied and includes mice, frogs, snakes, birds, and small mammals. A variety of virus infections have been identified in mink, including those spread by cross-species transmission. During the ongoing SARS-CoV-2 pandemic, infection of mink on two farms in the Netherlands was reported(Oreshkova et al., 2020). Mink has been a neglected mammalian host, despite being infected by many subtypes of influenza A viruses, including both mammalian and avian influenza A viruses(Zhao et al., 2019). From an epidemiological standpoint, minks may be an important sentinel species for surveillance and early warning of outbreaks of viral diseases. Despite this potential, there is limited knowledge of the dynamics of cross-species virus transmission in the models in relevant settings. Most studies have relied on prospective inference and reconstruction of infections, without adequate knowledge of the mechanism of immunity.

Here, we studied SVV isolated from a farm that was the site of SVV infection. The associated clinical signs and pathological and virological findings are described. Sequence analysis of SVV implicated the virus as a probable source of the initial infection. Transmission of SVV between mink, mice, and pigs may occur following excretion by the mink and subsequent environmental persistence of the virus. The data presented in this study are the first description of the cross-species transmission of SVV in mink.

\section{MATERIALS AND METHODS}

\subsection{Ethics statement}

This study was conducted following with the animal welfare guidelines of the World Organization for Animal 
Health. All of the clinical animal samples used in this study were approved by the Committee on Ethics of the Animal Science and Technology College of Northeast Agricultural University, for routine testing. The animal health codes used was NEAU201918.

\section{2}

\section{Cell reagents and virus isolation}

Baby hamster kidney 21 (BHK-21) cells were cultured in Dulbecco's modified Eagle's medium (DMEM; Invitrogen, Carlsbad, CA, USA) at $37^{\circ} \mathrm{C}$ in a humidified incubator in a $5 \% \mathrm{CO}_{2}$ atmosphere. The cells were used for the proliferation of SVV recovered from tissue samples of infected piglets on a farm in Heilongjiang Province. SVV viral RNA was extracted from fecal samples and was converted to cDNA by using reverse transcriptase HiScript @Q RT SuperMix (+gDNA wiper) and synthetic cDNA primers (Vazyme, Nanjing, China) (J. Zhang et al., 2019). cDNA was used as a follow-up template for PCR analysis of SVV-specific primers. The purified PCR products were sequenced (GenScript, Nanjing, China). The SVV-1/2 primer was used to amplify the VP1 of SVV. Other vesicular disease viruses, such as foot-and-mouth disease virus (FMDV, serotypes Asia 1, O, and A), Vesicular stomatitis virus (VSV), swine vesicular disease virus (SVDV), and Vesicular exanthema of swine virus (VESV) were amplified using RT-PCR. The virus isolation method was used with BHK-21 cells as previously reported(Leme et al., 2015). The cells were incubated for 2 days until a cytopathic effect (CPE) was observed(Yang et al., 2018) (Figure 1A). Cells displaying CPE were tested for SVV by RT-PCR as described above. The isolated strain was designated as SVV-CH-09-2018. SVV-CH-09-2018 was added to BHK-21 cells at a multiplicity of infection of 0.5 and 1 for one-step growth curves (Fernandes et al., 2018) (Figure 1B)

\subsection{Cell preparation for transmission electron microscopy (TEM)}

BHK-21 cells infected with SVV-CH-09-2018 for $18 \mathrm{~h}$, were washed twice with pre-cooled PBS and fixed with glutaraldehyde at $4^{\circ} \mathrm{C}$. The cells were recovered by scraping and were centrifuged at $12000 \mathrm{~g}$ for $10 \mathrm{~min}$. The supernatant was discarded. The cells were post-fixed in $\mathrm{OsO}_{4}$, embedded in epoxy resin, and polymerized at $80^{\circ} \mathrm{C}$ for at least 3 days. Finally, the cell samples were cut into 60 -nm slices and stained with uranyl acetate(Malenovska, 2013). The sections mounted on grids were observed by TEM, using a model HT7600 microscope (Hitachi, Japan).

\subsection{Sequencing and phylogenetic analyses}

The SVV-CH-09-2018 whole genome was divided into seven overlapping fragments. The fragments were PCR-amplified using Prime STAR@HS (TaKaRa Bio, Dalian, China). The primers are listed in Table 1. The PCR product was purified and cloned into the pEASY®-Blunt Simple clone vector (Transgene, China, and the cloned products were sequenced by Sangon Biotech (Shanghai, China). The SeqMan II program in the DNASTAR software package (DNASTAR, Madison, WI, USA) was used to assemble the seven overlapping fragment sequences into a complete genomic sequence, using the 5'-full RACE core kit and 3'-full RACE core kit (TaKaRa Bio). Specific primers were used to detect 5'- and 3'-untranslated regions (UTRs) (Table 1). The sequences targeted by the primers were designed using the existing SVV sequence with the PCR product sequence.

To construct the gene development tree, we first analyzed the SVV-CH-09-2018 gene sequence by using BLAST(Leme, Alfieri, \& Alfieri, 2017). After obtaining and filtering similar sequences, we screened SVV genes from viruses isolated from several countries, including the United States, China, Canada, Vietnam, Colombia, and Brazil(Hales et al., 2008; Leme et al., 2015). MEGA7.0 and OMICSTUDIO evolutionary tree software were used for the phylogenetic genome analysis, after aligning the selected block by Clustal, using the Neighbor-Joining method. In the phylogeny test, we used the Bootstrap method with 1000 replications. Evolutionary tree processing was continued in OMICStudio after the preliminary evolutionary tree was obtained.

\subsection{Mink challenge assay}


The SVV-CH-09-2018 strain was used for the challenge test. Mink $(n=6)$ weaned for approximately 60 days were observed for 1 week to ensure they were asymptomatic. SVV, FMDV, SVDV, VSV, and pseudorabies virus were not detected by the corresponding ELISA and RT-PCR or PCR(Luis Gabriel Gimenez-Lirola et al., 2016). The mink were divided into two groups, the 3 minks were in each group. The first group was injected intraperitoneally with a $5 \mathrm{~mL}$ volume of strain SVV-CH-09-2018 $\left(1 \times 10^{9}\right.$ median cell culture infective dose [TCID50]/mL). The second group was inoculated with DMEM as a negative control (NC). Both groups were fed under the same conditions and in separate rooms. Strict biosafety protocols were followed to avoid crossover contamination. Clinical symptoms were monitored daily for 28 days. At $0,3,7$, 14,21 , or 28 days post-challenge ( $\mathrm{dpc}$ ), serum was collected from each animal, and the anti-SVV neutralizing antibody titer was determined(Joshi, Fernandes, et al., 2016). TaqMan real-time RT-PCR was used to detect viral load(Dall Agnol et al., 2017) (J. Zhang et al., 2019). A standard curve was generated by plotting the threshold values against serially diluted plasmid DNA encoding the SVV VP1 gene fragment. At 28 dpc, the mice were euthanized for pathological examination. The heart, spleen, liver, kidney, lung, inguinal lymph nodes, and other organs were collected for histopathological observation. TaqMan real-time RT-PCR was used to detect mRNA viral titers in these organs.

\subsection{Pathological and immunofluorescence examinations}

Intestinal tissues were collected for the examinations within 10 to 15 min after the death of each mink. Each tissue sample was impregnated with formalin by soaking in $10 \%$ formalin for more than $24 \mathrm{~h}$ at room temperature, and the samples were then soaked in different concentrations of ethanol from $100 \%$ to $70 \%$ for $2 \mathrm{~h}$. The samples were then embedded in paraffin wax and sectioned $(6 \mu \mathrm{m})$. The sections were stained with hematoxylin and eosin for histological examination using light microscopy (Olympus, Tokyo, Japan). The dewaxing sections were used for immunofluorescence assay(Hou et al., 2018). The sections were blocked with $0.3 \%$ bovine serum albumin in PBS at room temperature (RT) for $3 \mathrm{~h}$ and then washed three times with pre-cooled PBS. This was followed by permeabilization with $0.4 \%$ Triton X-100 at RT, after which, the sections were incubated for 45 min at RT with anti-SVV VP1 antibody, which was synthesized in our lab. The sections were then labeled with secondary goat anti-mouse IgG H\&L antibody (FITC) (Abcam, Cambridge, UK) for $30 \mathrm{~min}$ at RT. Finally, the samples were washed with PBS, and examined by fluorescence microscope (Leica, Wetzlar, Germany).

\subsection{Production of specific SVV antibody}

All mink were fed in the same manner. Clinical symptoms were monitored daily for 28 days. At $0,3,7$, 14,21 , or $28 \mathrm{dpc}$, mink serum was collected to examine the quality of specific antibodies by ELISA. We expressed the VP1 protein in a prokaryotic system. The secondary horseradish peroxidase-conjugated (HRP) rabbit anti-mink IgG antibody (SolarBio, Beijing, China) was added, and then incubated for $1 \mathrm{~h}$ at RT, and then washed three times; o-Phenylenediamine dihydrochloride was used as the chromogen. The absorbance was measured at $490 \mathrm{~nm}$ by using an ELx800 microplate reader (BioTek Instruments, Winooski, VT, USA). The results of each group of plates were standardized using a panel of reference IgG negatives and positives. Positive/negative $(\mathrm{P} / \mathrm{N})$ ratios $>2$ were considered positive.

\subsection{Statistical analyses}

Data between the different groups were determined with one-way repeated-measures analysis of variance (ANOVA) and the least significance difference (LSD) test. Differences were considered statistically significant at $\mathrm{p}<0.05$.

\section{RESULTS}

\subsection{Isolation and purification of the SVV strain}

RT-PCR was not designated to detect Teschovirus A, Sapelovirus A, Enterovirus G, FMD, VS, and SVD virus in the vesicular outbreak clinical samples. The expected 542-bp product of SSV was detected in all vesicular fluid swabs from the pigs. After two sequential passages in BHK-21 cells, SVV was successfully isolated from the vesicular fluid samples. A cytopathic effect was evident 2 days following the addition of 
SVV to BHK-21 cell cultures. The changes were not observed in the NC (Figure 1A). RT-PCR detected only SSV. FMD, VS, SVD, Teschovirus A, Sapelovirus A, and Enterovirus G were not detected in the infected BHK-21 cells. A one-step growth curve of SVV-CH-09-2018 in BHK-21 cells was performed at a multiplicity of infection of 0.1 and 0.5 . The infected cells were collected at $0,4,8,12,16,20,24,28,32$, and $36 \mathrm{~h}$ postinfection (hpi) for determination of TCID50. The virus began to quickly replicate at $4 \mathrm{hpi}$, with the highest titers at $32 \mathrm{hpi}$. The maximum viral titer was $2.59 \times 10^{9}$ at $32 \mathrm{hpi}$ (Figure 1B). At $24 \mathrm{hpi} \mathrm{BHK-21}$ cells were examined by electron microscopy. The virions observed were round with a diameter of approximately $30 \mathrm{~nm}$ (Figure 1A).

\subsection{SVV-CH-09-2018 sequence and evolutionary analysis}

An evolutionary tree was drawn and analyzed using MEGA7.0 and OMICSTUDIO evolutionary tree software. A genome schema map (Figure 2) was constructed based on prototype SVV-CH-09-2018 isolated by our lab. The general structure of the genome included leader protein, 5' and 3' UTRs, P1 region proteins (capsid proteins), P2 region proteins (nonstructural proteins), and P3 region proteins (nonstructural proteins). The evolutionary tree revealed that SVV-CH-09-2018 had the typical L-4 genome layout of picornaviruses. A previous phylogenetic analysis showed that SVV strains could also be divided into four branches. The SVVCH-09-2018 strain belonged to clade III; it shared the highest homology with the American Senecavirus A strain HB-CH-2016 (GenBank, KX377924.1; 99.66\%) and the Senecavirus A strain CH-01-2015 isolated in China (GenBank, KT321458.1; 99.67\%).

The evolutionary tree revealed the presence of Seneca genes from isolates from the United States, China, Canada, Vietnam, Colombia, and Brazil, indicating the global distribution of the virus. The relationship of various strains isolated from different countries is indicated in Figure 2A with different colors used to denote different strains. The spread of global SVV genomes was evident, with three major evolutionary clusters: United States, China, and Canada-like strain clusters. To date, more than half of the SVVs in China was similar with SVV from the US, and a part of the SVVs in China were from the US and Canada strains. To shed more light on how the Seneca virus is spreading globally, we compiled an SSV chronology of landmark incidents. The chronology was combined with the evolutionary tree to facilitate the analysis of the evolution and prevalence of the Seneca molecular epidemiology of SVV. As shown in Figure 2 and Table 2, the strains from different locations or dates, were separated, which implies that the geographic distribution and infectious host may contribute to the codon usage pattern in the evolution of SVA. The geographic distribution and host are the two main mutational-pressures of natural selection. The origin of the strain that was the focus of the present study, was not clear. The collective data favored the view that the pathogenicity of SSV has increased since 2015, with increased morbidity and mortality rates associated with the infections. The strain that we have examined has been continuously evolving in China for some time. Further investigations into the evolution and prevalence of SVV, including identification of the pathogenesis and molecular epidemiology, are urgently needed.

\subsection{SVV mink infection}

Five-day challenge experiments in mink did not result in any deaths. The pathological results did not indicate frequent gross changes, such as alteration of the surface of the lungs, ulcerative lesions, and lesions of the liver and kidney. However, liquid feces were evident on the second day of SVV infection, which indicated diarrhea in the small intestine. Examination of all tissues for the presence of SVV nucleic acid, and the RTPCR and qRT-PCR results showed that two of three oral-fluid samples were positive, and all three minks of fecal-swab samples were tested with positive. No SVV nucleic acid was detected in the serum of infected minks. qRT-PCR detection of SVV RNA yielded results corresponding to the RT-PCR SVV nucleic acid levels. However, fecal-swab samples of the infected minks contained a markedly higher number of RNA copies than the oral-fluid samples. No samples in the control group tested positive, upon RT-PCR and qRT-PCR (Table 3).

\subsection{Pathological examination and Immunofluorescence of SVV infection}

Microscopic examination revealed obvious differences among these groups of minks concerning to their small- 
intestine samples obtained from different intestines. The principal histopathological results are graphically summarized in Figure 3A. In the intestinal sections, lesions were observed in the duodenum and colon. The principal observations were atrophy and rupture, followed by fusion of villi in the duodenum and colon. Inflammation was observed in different intestinal segments. Inflammation in the duodenum of the infected mink was much more severe compared to that observed in the control group. Besides, necrosis and vacuolization of epithelial cells were evident in the minks with clinical manifestations of diarrhea. The duodenum and colon sections of the control group mink did not show any pathological changes compared with the infectious group.

The distribution and quantity of SVV virions in the small intestine of the mink are shown in Figure 3B. SVV virion antigens were identified by the VP1 multi-antibody produced in our lab. Viral antigens were mainly detected in villous epithelial cells in both the duodenum and colon. The antigens were not present in the duodenum or colon of the control group. However, viral antigens were detected in the duodenum and colon of infected mink, and different intestinal segments contained different virus titers. The colon had much higher titers than the duodenum in SVV infection, indicating that the epithelial cells of the colon were much more sensitive to the infection than the epithelial cells of the duodenum.

\subsection{Quality of $S V V$-specific antibody}

All minks were fed in the same manner. Clinical symptoms were monitored daily for 28 days. At 0, 7, 14, 21 , or $28 \mathrm{dpc}$, serum was collected to measure the anti-SVV neutralizing antibody titer in the two groups (Figure 3C). SVV antibodies were detected in the infected group and their change was measured over the 28-day experiment. No change in antibody detection or levels was observed in the control group. In infected mink, the antibodies gradually increased from the first to the third week. The antibody titer peaked during the third week and then decreased slightly but non-significantly.

\section{DISSCUSSION}

Senecavirus A is a vesicular disease in pigs. The disease, also known as porcine idiopathic vesicular disease, is important for the health of the animals and thereby, the farm economy. Recent SVV outbreaks have been reported in many countries with large swine production, similar to outbreaks of other important vesicular viruses, including VSV, SVDV, and FMDV(Canning et al., 2016). SVV has received much attention, with a focus on virus pathogenesis, immunology, and epidemiology(Segalés, Barcellos, Alfieri, Burrough, \& Marthaler, 2016). The present study has clarified the characteristics of the pathogenesis of SVV infection in epithelial and epidermal cells, immunosuppression, immune evasion, and cross-host transmission.

In 2015, the first outbreak of vesicular lesions in newborn piglets was observed at farms in Guangdong Province of China(H. Zhang et al., 2020). The cause of the high-mortality outbreak was identified as SVA infection. Since then, more than half of the province has been affected by SVV infection. The strain isolated in the present study is the first to be reported from the northernmost province of China. SVV has first identified 30 years ago since it was first reported in the US. A turning point like of the outbreaks occurred in 2015, after which, several outbreaks of SVV vesicular disease (SVA-VD) and epidemic transient neonatal loss occurred(Canning et al., 2016; Zhu et al., 2017). Prior to 2010, isolates were not pathogenic and did not display clinical signs. Strains isolated after 2015 are considered accompanied by the vesicular lesion. Our phylogenetic comparison of recent SVA isolates with isolates obtained before 2010 revealed a marked divergence of 5.59\%(Saeng-Chuto, Stott, et al., 2018). Therefore, SVV strains isolated before 2010 are considered "historical" (Houston, Temeeyasen, \& Pineyro, 2020). Seneca Valley virus-1 (SVV-001) was first detected in a PER.C6 fetal retinoblast cell culture in 2002. It was likely a contaminant from the bovine serum or porcine trypsin used in the cell culture(Venkataraman et al., 2008). SVVs have experienced great change in their nucleotide composition over the past ten years and have been identified in different hosts and tumor cells. Mutational pressure from several animal hosts accelerates the frequency of recombinant mutations in SVV(Canuti et al., 2020). Cross-host transmission may have led to a rapid increase in the rate at which mutant stress has an effect.

The pathogenesis of different types of US strains of SVV varies in pigs, even though they have similar 
sequences(H. Zhang et al., 2020). However, the replication efficiencies of the different strains were all similarly high. These characteristics imply that SVVs have the potential to infect various host animals. Notably, SVVs have been detected and isolated from pigs, environmental samples, mouse feces, and mouse small-intestine, and SVV RNA was also detected in houseflies from farms that were negative for SVV vesicular disease(Joshi, Mohr, et al., 2016). A 2012 report described the occurrence of SVV accompanied by vesicular lesions and a spontaneous outbreak from a pig purchased at the Indiana State Fair(Leme et al., 2017). In 2015, SVV was first detected in China, an outbreak occurred in 2016. The three major evolutionary clusters have been identified in China, as compared to the US and Canada(Zhu et al., 2017). Additionally, all Chinese isolates could be grouped into clusters present in the US and Canada. As shown in Figure 2A, the isolated strain mainly belonged to the US-like cluster.

Mink were infected with SVV in our research, the virus was detected in oral fluid and fecal-swab samples by RT-PCR and qRT-PCR, which indicated that the mink was an important SVV host. Mink was the fourth most frequently infected host, following humans, swine, and mice(Feronato et al., 2018). The qRT-PCR results indicated that fecal swabs had a much higher quality SVV mRNA than the oral fluid. The pathogenesis and clinical data revealed pathologic changes in the intestinal tract. No vesicular lesions were observed in SVV infected mink. Histologically, piglets had multifocal pathological changes, such as infiltration of inflammatory cells, necrotic keratinocytes, and hemorrhage(Leme et al., 2016). Clinical evaluation in finisher pigs also showed that the virus can present subclinical signs or no clinical signs. In experimentally infected pigs, infiltration of inflammatory cells and necrotic keratinocytes were evident. In addition, the histopathologic lesions in the piglets were more serious than those in the piglets, and they were accompanied by interstitial pneumonia and ballooning degeneration of the urinary bladder and renal pelvis epithelium(Leme et al., 2016). All of these histopathologic changes indicate that the SVV can invade the epithelium and epidermis cells in mammals, such as pigs and minks. However, we still do not clearly understand the mechanism of SVV infection of intestinal epithelial cells and oral epithelial cells in mink.

The risk of SVA infection varies markedly between herds and farms. Risk factors including a high number of breeding females, a higher number of farm employees, and the time of weaning may contribute to the spread of SVV(Tousignant et al., 2017). A serology analysis in animals detected neutralizing antibodies to SVV in 27 of 71 porcine samples, 10 of 30 bovine samples, and five of 35 wild mouse samples, with no neutralizing antibodies detected in more than 100 human serum samples(Reddy et al., 2007). Taken together, these data show that SVV could naturally replicate in farm animals and humans, and that farm animal could be stimulated to produce neutralizing antibodies(Baker et al., 2017). In contrast, the production of neutralizing antibodies in humans is relatively rare. Virus shedding could be detected up to 28 days post-infection. However, persistent shedding of the virus could be sustained up to 60 days following SVV infection(Maggioli et al., 2019). Finisher pigs reportedly produce neutralizing antibodies at 5 dpi following experimental inoculation, with maximum antibody concentrations between 7 and 14 dpi. However, neutralizing antibodies decreased incrementally during the first two weeks post-infection(Houston et al., 2020). In a longitudinal study on SVA-infected farms, the antibody titers of piglets were higher during the first week of age, but disappeared in the last four and five weeks after born. More importantly, $20 \%$ to $40 \%$ of piglets with neutralizing antibodies presented viremia and viral shedding in feces and oral fluids, which were sustained between four and five weeks without clinical symptoms(Tousignant et al., 2017). Another study reported high SVA genetic diversity in samples collected over 12 months from swine and several sites in their environments(Joshi et al., 2020). The special immune and infection status exerted mutation pressure, which was the main driver of the evolution of SVV, rather than natural selection.

In the present study, SVV nucleic acid was detected from swabs of internal and external surfaces in the farm. The result indicates that SVV poses an environmental risk. The prior detection of SVA in mice and houseflies indicated that these may play a role in the epidemiology of SVV, which would also increase the risk of SVV infecting wild animals as natural hosts(Joshi, Mohr, et al., 2016). The latter may act as a natural reservoir and thus, a potential vector. Mink are higher in the food chain than mice (Figure4). Thus, it is possible that SVV could infect mink and that it could evolve. Mutational pressure has been considered the major factor in the variation, compared with natural selection. All the studies to date have focused on the 
role of geographic distribution in contributing to the codon usage pattern of SVA. Mutational pressure has a more important role in SVA evolution than natural selection(Chen et al., 2017). However, no study has focused on cross-species transmissions, such as the complex links between physiological differences in hosts, disease progression, and viral release. Mink infected with SVV provide a new avenue of mutational pressure. Studies of SVV infection in mink could increase the understanding of the cross-species transmission of SVV and the viral life cycle within the environment. The collective knowledge could inform the prevention of SVA infection.

SVV exhibits immune evasion activity in the immune system of humans and other mammals. Analyses involving antibodies to SVV surface antigens showed that SVV could stimulate the immune system of mink. Antibody titers increased with the infection of SVV in mink. In the clinical evaluation, there were no differences in the level of the IgG antibody dynamics between clinically affected and non-affected animals. Immune evasion was the major characters in the identification of SVV as a potent oncolytic virus against tumors in medicine; other features include the targeting and penetration of solid tumors following intravenous administration, the inability of insertional mutagenesis, and self-replication with selective tropism for cancer cells(Burke, 2016). A strong cellular immune response was reportedly induced by SVV infection, which promotes the response of interferon-gamma -specific T cells as early as 3-7 dpi(L. G. Gimenez-Lirola et al., 2016). T-cell responses did not completely clear SVV at the first 14 dpi. However, the evolution of SVV from the same infecting farm for one year indicated that the evolution was not from a single host, in this case of the pig. Likely, the multiple hosts are the mutational pressure promoting the SVV evolution and cross-species transmission.

\section{CONFLICT OF INTEREST STATEMENT}

The authors declare no financial or commercial conflicts of interest.

\section{ACKNOWLEDGEMENTS}

This work was supported by the National Natural Science Foundation of China (Grant number:31902169), the Heilongjiang Province Science Fund for Excellent Young Scholars (Grant number:YQ2020C008),Young Talents" Project of Northeast Agricultural University(Grant number:18QC39)and Student Innovation Practical Training of NEAU.

\section{REFERENCE}

Adams, M. J., Lefkowitz, E. J., King, A. M., Bamford, D. H., Breitbart, M., Davison, A. J., . . . Carstens, E. B. (2015). Ratification vote on taxonomic proposals to the International Committee on Taxonomy of Viruses (2015). Arch Virol, 160 (7), 1837-1850. doi:10.1007/s00705-015-2425-z

Arzt, J., Bertram, M. R., Vu, L. T., Pauszek, S. J., Hartwig, E. J., Smoliga, G. R., . . . Dung, D. H. (2019). First Detection and Genome Sequence of Senecavirus A in Vietnam. Microbiol Resour Announc, 8 (3). doi:10.1128/MRA.01247-18

Baker, K. L., Mowrer, C., Canon, A., Linhares, D. C., Rademacher, C., Karriker, L. A., \& Holtkamp, D. J. (2017). Systematic Epidemiological Investigations of Cases of Senecavirus A in US Swine Breeding Herds. Transbound Emerg Dis, 64 (1), 11-18. doi:10.1111/tbed.12598

Bo-Shun, Z., Li, L. J., Qian, Z., Zhen, W., Peng, Y., Guo-Dong, Z., . . . Zhi-Jing, X. (2020). Co-infection of H9N2 influenza virus and Pseudomonas aeruginosa contributes to the development of hemorrhagic pneumonia in mink. Vet Microbiol, 240 , 108542. doi:10.1016/j.vetmic.2019.108542

Burke, M. J. (2016). Oncolytic Seneca Valley Virus: past perspectives and future directions. Oncolytic Virother, 5 , 81-89. doi:10.2147/OV.S96915

Burke, M. J., Ahern, C., Weigel, B. J., Poirier, J. T., Rudin, C. M., Chen, Y., . . . Blaney, S. M. (2015). Phase I trial of Seneca Valley Virus (NTX-010) in children with relapsed/refractory solid tumors: a report of the Children's Oncology Group. Pediatr Blood Cancer, 62 (5), 743-750. doi:10.1002/pbc.25269 
Canning, P., Canon, A., Bates, J. L., Gerardy, K., Linhares, D. C., Pineyro, P. E., . . . Karriker, L. (2016). Neonatal Mortality, Vesicular Lesions and Lameness Associated with Senecavirus A in a U.S. Sow Farm. Transbound Emerg Dis, 63 (4), 373-378. doi:10.1111/tbed.12516

Canuti, M., Todd, M., Monteiro, P., Van Osch, K., Weir, R., Schwantje, H., . . . Lang, A. S. (2020). Ecology and Infection Dynamics of Multi-Host Amdoparvoviral and Protoparvoviral Carnivore Pathogens.Pathogens, 9 (2). doi:10.3390/pathogens9020124

Chen, Y., Xu, Q., Tan, C., Li, X., Chi, X., Cai, B., . . . Chen, J. L. (2017). Genomic analysis of codon usage shows influence of mutation pressure, natural selection, and host features on Senecavirus A evolution. Microb Pathog, 112 , 313-319. doi:10.1016/j.micpath.2017.09.040

Dall Agnol, A. M., Miyabe, F. M., Leme, R. A., Oliveira, T. E. S., Headley, S. A., Alfieri, A. A., \& Alfieri, A. F. (2017). Quantitative analysis of senecavirus A in tissue samples from naturally infected newborn piglets. Archives of Virology, 163 (2), 527-531. doi:10.1007/s00705-017-3630-8

Fernandes, M. H. V., Maggioli, M. F., Joshi, L. R., Clement, T., Faccin, T. C., Rauh, R., . . . Diel, D. G. (2018). Pathogenicity and cross-reactive immune responses of a historical and a contemporary Senecavirus A strains in pigs. Virology, 522 , 147-157. doi:10.1016/j.virol.2018.06.003

Feronato, C., Leme, R. A., Diniz, J. A., Agnol, A. M. D., Alfieri, A. F., \& Alfieri, A. A. (2018). Development and evaluation of a nested-PCR assay for Senecavirus A diagnosis. Trop Anim Health Prod, 50 (2), 337-344. doi:10.1007/s11250-017-1436-z

Gimenez-Lirola, L. G., Rademacher, C., Linhares, D., Harmon, K., Rotolo, M., Sun, Y., . . . Pineyro, P. (2016). Serological and Molecular Detection of Senecavirus A Associated with an Outbreak of Swine Idiopathic Vesicular Disease and Neonatal Mortality. J Clin Microbiol, 54 (8), 2082-2089. doi:10.1128/JCM.00710-16

Gimenez-Lirola, L. G., Rademacher, C., Linhares, D., Harmon, K., Rotolo, M., Sun, Y., . . . Fenwick, B. W. (2016). Serological and Molecular Detection of Senecavirus A Associated with an Outbreak of Swine Idiopathic Vesicular Disease and Neonatal Mortality. Journal of Clinical Microbiology, 54 (8), 2082-2089. doi:10.1128/jcm.00710-16

Hales, L. M., Knowles, N. J., Reddy, P. S., Xu, L., Hay, C., \& Hallenbeck, P. L. (2008). Complete genome sequence analysis of Seneca Valley virus-001, a novel oncolytic picornavirus. J Gen Virol, 89 (Pt 5), 12651275. doi:10.1099/vir.0.83570-0

Hou, X., Jiang, X., Jiang, Y., Tang, L., Xu, Y., Qiao, X., . . . Li, Y. (2018). Oral Immunization against PEDV with Recombinant Lactobacillus casei Expressing Dendritic Cell-Targeting Peptide Fusing COE Protein of PEDV in Piglets. Viruses, 10 (3). doi:10.3390/v10030106

Houston, E., Temeeyasen, G., \& Pineyro, P. E. (2020). Comprehensive review on immunopathogenesis, diagnostic and epidemiology of Senecavirus A. Virus Res, 286 , 198038. doi:10.1016/j.virusres.2020.198038

Jayawardena, N., Burga, L. N., Easingwood, R. A., Takizawa, Y., Wolf, M., \& Bostina, M. (2018). Structural basis for anthrax toxin receptor 1 recognition by Seneca Valley Virus. Proc Natl Acad Sci U S A, 115 (46), E10934-E10940. doi:10.1073/pnas.1810664115

Joshi, L. R., Fernandes, M. H. V., Clement, T., Lawson, S., Pillatzki, A., Resende, T. P., . . . Diel, D. G. (2016). Pathogenesis of Senecavirus A infection in finishing pigs. J Gen Virol, 97 (12), 3267-3279. doi:10.1099/jgv.0.000631

Joshi, L. R., Mohr, K. A., Clement, T., Hain, K. S., Myers, B., Yaros, J., . . . Diel, D. G. (2016). Detection of the Emerging Picornavirus Senecavirus A in Pigs, Mice, and Houseflies. J Clin Microbiol, 54 (6), 1536-1545. doi:10.1128/JCM.03390-15 
Joshi, L. R., Mohr, K. A., Gava, D., Kutish, G., Buysse, A. S., Vannucci, F. A., . . . Diel, D. G. (2020). Genetic diversity and evolution of the emerging picornavirus Senecavirus A. J Gen Virol, 101 (2), 175-187. doi:10.1099/jgv.0.001360

Leme, R. A., Alfieri, A. F., \& Alfieri, A. A. (2017). Update on Senecavirus Infection in Pigs. Viruses, 9 (7). doi:10.3390/v9070170

Leme, R. A., Miyabe, F. M., Dall Agnol, A. M., Alfieri, A. F., \& Alfieri, A. A. (2019a). A new wave of Seneca Valley virus outbreaks in Brazil. Transbound Emerg Dis, 66 (3), 1101-1104. doi:10.1111/tbed.13151

Leme, R. A., Miyabe, F. M., Dall Agnol, A. M., Alfieri, A. F., \& Alfieri, A. A. (2019b). Seneca Valley virus RNA detection in pig feed and feed ingredients in Brazil. Transbound Emerg Dis, 66 (4), 1449-1453. doi:10.1111/tbed.13215

Leme, R. A., Oliveira, T. E. S., Alfieri, A. F., Headley, S. A., \& Alfieri, A. A. (2016). Pathological, Immunohistochemical and Molecular Findings Associated with Senecavirus A-Induced Lesions in Neonatal Piglets. J Comp Pathol, 155 (2-3), 145-155. doi:10.1016/j.jcpa.2016.06.011

Leme, R. A., Zotti, E., Alcantara, B. K., Oliveira, M. V., Freitas, L. A., Alfieri, A. F., \& Alfieri, A. A. (2015). Senecavirus A: An Emerging Vesicular Infection in Brazilian Pig Herds. Transbound Emerg Dis, 62 (6), 603-611. doi:10.1111/tbed.12430

Liu, J., Li, Z., Cui, Y., Yang, H., Shan, H., \& Zhang, C. (2020). Emergence of an Eurasian avian-like swine influenza A (H1N1) virus from mink in China. Vet Microbiol, 240 , 108509. doi:10.1016/j.vetmic.2019.108509

Maggioli, M. F., Fernandes, M. H. V., Joshi, L. R., Sharma, B., Tweet, M. M., Noll, J. C. G., . . . Diel, D. G. (2019). Persistent Infection and Transmission of Senecavirus A from Carrier Sows to Contact Piglets. $J$ Virol, 93 (21). doi:10.1128/JVI.00819-19

Malenovska, H. (2013). Virus quantitation by transmission electron microscopy, TCID50, and the role of timing virus harvesting: A case study of three animal viruses. Journal of Virological Methods, 191 (2), 136-140. doi:10.1016/j.jviromet.2013.04.008

Mombo, I. M., Berthet, N., Lukashev, A. N., Bleicker, T., Brunink, S., Leger, L., . . . Rougeron, V. (2015). First Detection of an Enterovirus C99 in a Captive Chimpanzee with Acute Flaccid Paralysis, from the Tchimpounga Chimpanzee Rehabilitation Center, Republic of Congo.PLoS One, 10 (8), e0136700. doi:10.1371/journal.pone.0136700

Oberste, M. S., Feeroz, M. M., Maher, K., Nix, W. A., Engel, G. A., Begum, S., . . . Jones-Engel, L. (2013). Naturally acquired picornavirus infections in primates at the Dhaka zoo. J Virol, 87 (1), 572-580. doi:10.1128/JVI.00838-12

Omondi, G., Alkhamis, M. A., Obanda, V., Gakuya, F., Sangula, A., Pauszek, S., . . . VanderWaal, K. (2019). Phylogeographical and cross-species transmission dynamics of SAT1 and SAT2 foot-and-mouth disease virus in Eastern Africa. Mol Ecol, 28 (11), 2903-2916. doi:10.1111/mec.15125

Oreshkova, N., Molenaar, R. J., Vreman, S., Harders, F., Oude Munnink, B. B., Hakze-van der Honing, R. W., . . . Stegeman, A. (2020). SARS-CoV-2 infection in farmed minks, the Netherlands, April and May 2020. Euro Surveill, 25 (23). doi:10.2807/1560-7917.ES.2020.25.23.2001005

Pasma, T., Davidson, S., \& Shaw, S. L. (2008). Idiopathic vesicular disease in swine in Manitoba. Can Vet $J, 49(1), 84-85$.

Reddy, P. S., Burroughs, K. D., Hales, L. M., Ganesh, S., Jones, B. H., Idamakanti, N., . . . Hallenbeck, P. L. (2007). Seneca Valley virus, a systemically deliverable oncolytic picornavirus, and the treatment of neuroendocrine cancers. J Natl Cancer Inst, 99 (21), 1623-1633. doi:10.1093/jnci/djm198

Saeng-Chuto, K., Rodtian, P., Temeeyasen, G., Wegner, M., \& Nilubol, D. (2018). The first detection of Senecavirus A in pigs in Thailand, 2016. Transbound Emerg Dis, 65 (1), 285-288. doi:10.1111/tbed.12654 
Saeng-Chuto, K., Stott, C. J., Wegner, M., Kaewprommal, P., Piriyapongsa, J., \& Nilubol, D. (2018). The full-length genome characterization, genetic diversity and evolutionary analyses of Senecavirus A isolated in Thailand in 2016. Infect Genet Evol, 64 , 32-45. doi:10.1016/j.meegid.2018.06.011

Segales, J., Barcellos, D., Alfieri, A., Burrough, E., \& Marthaler, D. (2016). Senecavirus A. Veterinary Pathology, 54 (1), 11-21. doi:10.1177/0300985816653990

Sharma, B., Fernandes, M. H. V., de Lima, M., Joshi, L. R., Lawson, S., \& Diel, D. G. (2019). A Novel Live Attenuated Vaccine Candidate Protects Against Heterologous Senecavirus A Challenge. Front Immunol, 10 , 2660. doi:10.3389/fimmu.2019.02660

Stott, C. J., Temeeyasen, G., Tripipat, T., Kaewprommal, P., Tantituvanont, A., Piriyapongsa, J., \& Nilubol, D. (2017). Evolutionary and epidemiological analyses based on spike genes of porcine epidemic diarrhea virus circulating in Thailand in 2008-2015. Infect Genet Evol, 50 , 70-76. doi:10.1016/j.meegid.2017.02.014

Sun, D., Vannucci, F., Knutson, T. P., Corzo, C., \& Marthaler, D. G. (2017). Emergence and whole-genome sequence of Senecavirus A in Colombia. Transbound Emerg Dis, 64 (5), 1346-1349. doi:10.1111/tbed.12669

Tousignant, S. J. P., Bruner, L., Schwartz, J., Vannucci, F., Rossow, S., \& Marthaler, D. G. (2017). Longitudinal study of Senecavirus a shedding in sows and piglets on a single United States farm during an outbreak of vesicular disease. BMC Vet Res, 13 (1), 277. doi:10.1186/s12917-017-1172-7

Venkataraman, S., Reddy, S. P., Loo, J., Idamakanti, N., Hallenbeck, P. L., \& Reddy, V. S. (2008). Structure of Seneca Valley Virus-001: an oncolytic picornavirus representing a new genus. Structure, 16 (10), 15551561. doi:10.1016/j.str.2008.07.013

Wu, Q., Zhao, X., Bai, Y., Sun, B., Xie, Q., \& Ma, J. (2017). The First Identification and Complete Genome of Senecavirus A Affecting Pig with Idiopathic Vesicular Disease in China. Transbound Emerg Dis, 64 (5), 1633-1640. doi:10.1111/tbed.12557

Yang, F., Zhu, Z., Cao, W., Liu, H., Zhang, K., Tian, H., . . . Zheng, H. (2018). Immunogenicity and protective efficacy of an inactivated cell culture-derived Seneca Valley virus vaccine in pigs. Vaccine, 36 (6), 841-846. doi:10.1016/j.vaccine.2017.12.055

Zhang, H., Chen, P., Hao, G., Liu, W., Chen, H., Qian, P., \& Li, X. (2020). Comparison of the Pathogenicity of Two Different Branches of Senecavirus a Strain in China. Pathogens, 9 (1). doi:10.3390/pathogens9010039

Zhang, J., Nfon, C., Tsai, C. F., Lee, C. H., Fredericks, L., Chen, Q., . . . Lee, P. A. (2019). Development and evaluation of a real-time RT-PCR and a field-deployable RT-insulated isothermal PCR for the detection of Seneca Valley virus. BMC Vet Res, 15 (1), 168. doi:10.1186/s12917-019-1927-4

Zhao, P., Sun, L., Xiong, J., Wang, C., Chen, L., Yang, P., . . . Xiong, C. (2019). Semiaquatic mammals might be intermediate hosts to spread avian influenza viruses from avian to human. Sci Rep, 9 (1), 11641. doi:10.1038/s41598-019-48255-5

Zhu, Z., Yang, F., Chen, P., Liu, H., Cao, W., Zhang, K., . . . Zheng, H. (2017). Emergence of novel Seneca Valley virus strains in China, 2017. Transboundary and Emerging Diseases, 64 (4), 1024-1029. doi:10.1111/tbed.12662

\section{Figure Legend}

Figure 1. The process of BHK-21 cell CPEs induced by SVV-CH-09-2018 infecting cells (A) One-step growth curve of SVV-CH-09-2018.on BHK-21 cells with the multiplicity of infection (MOI) of 0.1 and 0.5 (B). An electron microscope image of the SVV virus $(\mathrm{C})$

Figure 2. The evolutionary trees are drawn by Mega 7.0 and Omicstudio, the analysis data were obtained from NCBI database with the whole genome of reference SVV strains. In the final evolutionary tree, the experimental virus strain SVV-CH has been marked 
(', andthestrains fromdif ferentcountriesarealsomarkedwithdif ferentcolor $(A)$.GenomestructureoftypetheSenecavirus, terminalnon-codingregion $\left(5^{\prime} U T R\right)$, a $3^{\prime}$-terminalnon-codingregion $\left(3^{\prime} U T R\right)$ encodingapolyprotein, andonlyoneopenreadi

Figure 3. The pathology and immunofluorescence indicated the pathological changes and the quality of virus virion. The pathological changes of duodenum and colon after the SVV infection (A). Viral antigens were detected mainly in villous epithelial cells of the colon. and the titer of the colon was much higher than that of the duodenum (B). In the mink challenge experiment, at $0,7,14,21$ and 28 days after challenge, mink sera were collected to determine the level of anti-SVA neutralizing antibody $(\mathrm{C})$.

Figure 4. To indicate the adaptation and transmission of SVV. An adaptation from pig SVV to human SVV includes two circulations, the food chain circulation and the reservoir circulation. In the reservoir and food chain circulation, SVV are transmitting, mutating, and adapting between mice and minks (as well as other semiaquatic mammals). Mink and mice can be infected through contacting with epidemic water. In a free stall barn system, usually in some areas of developing countries, pig will inevitably lead to a land habitat circulation including human beings. The blue pathway is transmitted by fecal route, while the red one is transmitted by oral route. In rural areas of South Asia, Southeast Asia, Southern and Eastern China, pigs mink and mice, in particular chick are often observed to eat by human. The pigs and mink also have the opportunity to eat mice feces, but mice seldom eat pig feces.

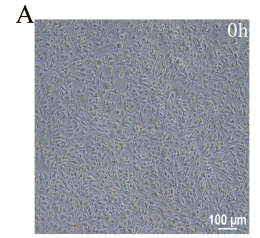

B

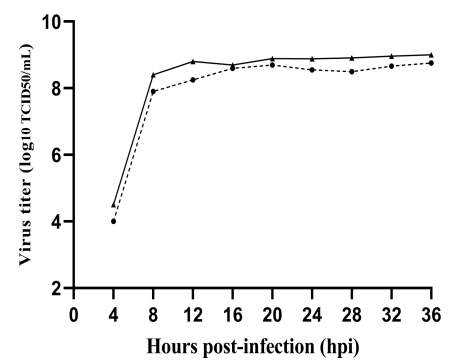

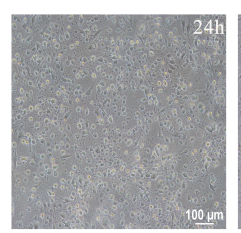

$\rightarrow 0.5 \mathrm{MOI}$

... $0.1 \mathrm{MOI}$
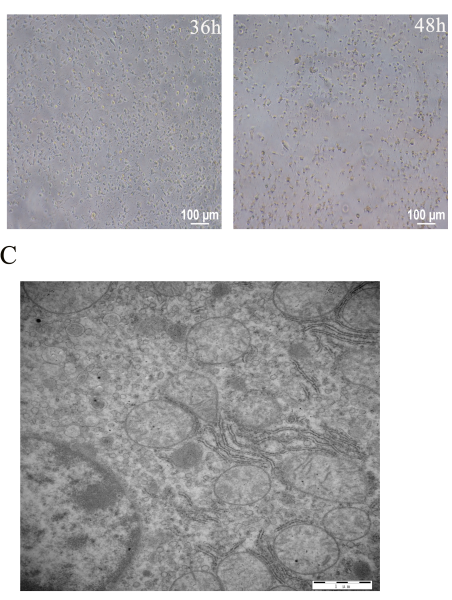


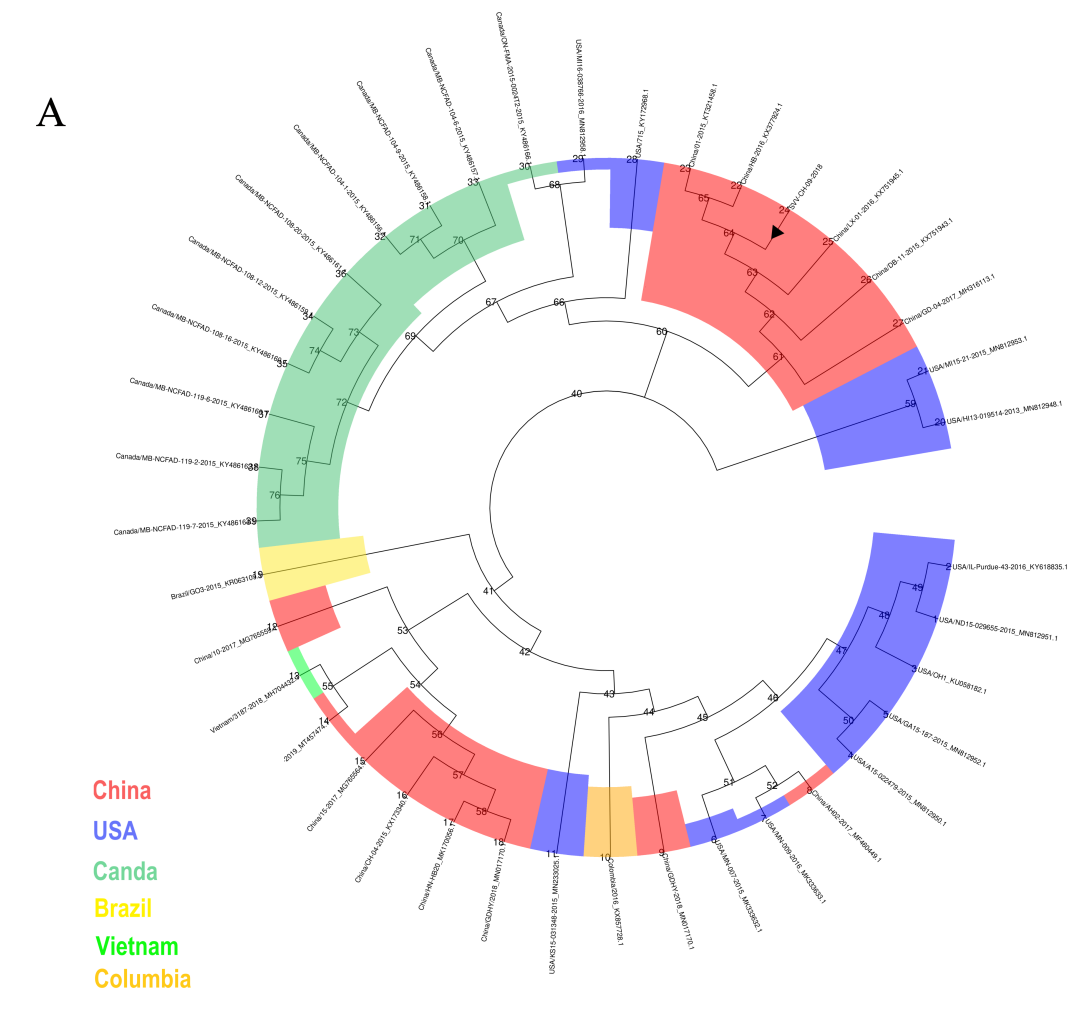

B

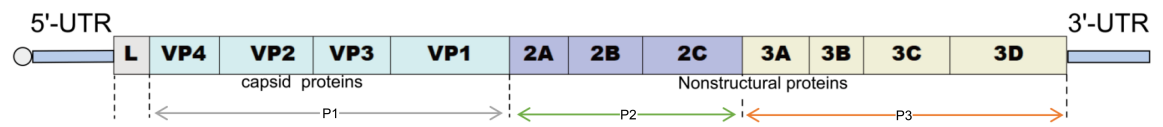


A

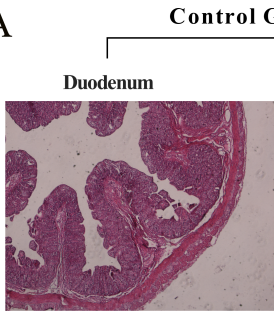

B
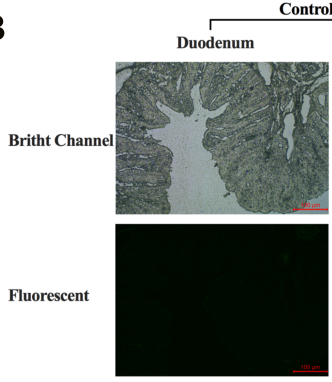

$\mathrm{C}$

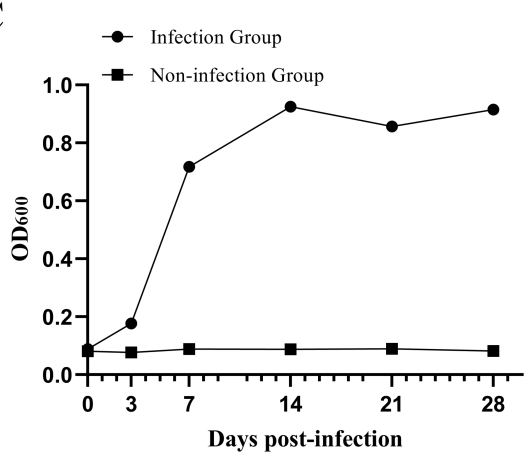

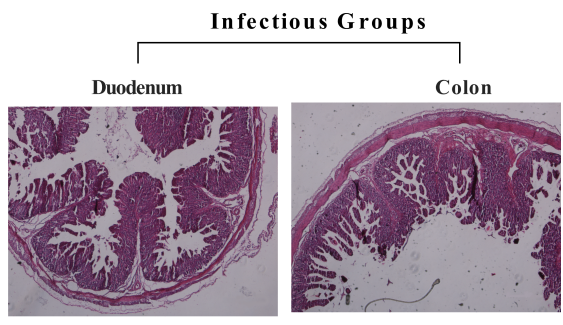
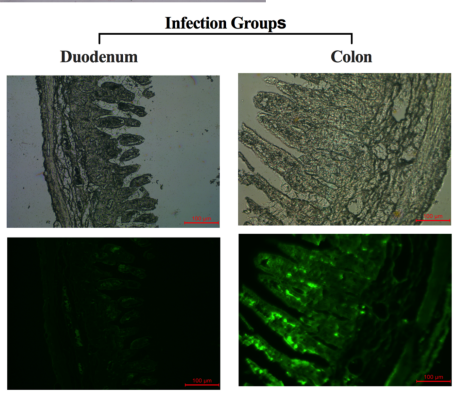

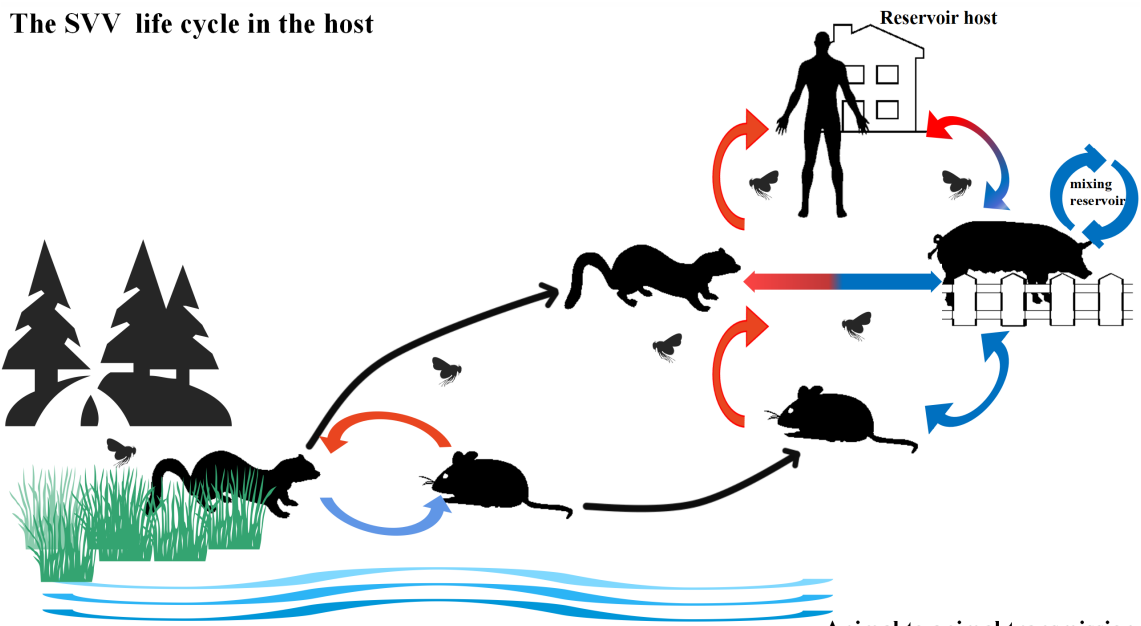

Hosted file

Table 1.pdf available at https://authorea.com/users/408482/articles/518474-isolation-andcharacterization-of-seneca-valley-virus-spread-from-pig-to-mink

Hosted file 
Table 2.pdf available at https://authorea.com/users/408482/articles/518474-isolation-andcharacterization-of-seneca-valley-virus-spread-from-pig-to-mink

\section{Hosted file}

Table 3.pdf available at https://authorea.com/users/408482/articles/518474-isolation-andcharacterization-of-seneca-valley-virus-spread-from-pig-to-mink 